\title{
Left Atrial Function After Electrical Conversion to Sinus Rhythm
}

\author{
H. IKRAM, P. G. F. NIXON, AND T. ARCAN \\ From the Cardiac Department, Charing Cross Hospital, London W.C.2
}

Atrial fibrillation is undesirable because it reduces the efficiency of the heart, particularly the diseased heart (Kory and Meneely, 1951) and gives rise to systemic or pulmonary embolism in about one-third of the cases (Goldman, 1960). The recent introduction of the use of the synchronized directcurrent shock (Lown, Amarasingham, and Neuman, 1962) has simplified the restoration of sinus rhythm, but little is known about the return of the mechanical activity to the atrium. Bramwell and Jones (1944) have suggested that an effective left atrial contraction may be absent in sinus rhythm, and cardiac catheterization studies have shown that the return of a $\mathbf{P}$ wave to the electrocardiogram may not be accompanied by the restoration of detectable mechanical activity to the left atrium (Braunwald, 1964; Logan et al., 1965). It is important to know if or when mechanical activity returns to the atrium; its failure to return may be a cause of continuing disability, and its delayed return may be associated with late embolism. Left heart catheterization is not a technique that is suitable for repeated use after conversion.

This paper describes a study of the left atrial contraction, by a simple bedside technique, in patients recently converted to sinus rhythm.

\section{SubJeCtS AND METHODS}

Fourteen patients were studied. Each had atrial fibrillation of at least five years' standing. The cause of fibrillation was rheumatic valvular heart disease in eight, atrial septal defect in two, ischaemic heart disease in one, thyrotoxicosis in two, and in one it was idiopathic.

In 12 patients the displacement curves of the skin overlying the apex of the left ventricle (ADC) were recorded simultaneously with a phonocardiogram by the method previously described by Nixon, Hepburn, and Ikram (1964). This technique is sufficiently sensitive to identify the small atrial or " $a$ " wave of healthy subjects (Fig. 1), and the enlarged " $a$ " wave of heart disease

Received October 12, 1966.
(Fig. 2). The relation of this " $a$ " wave to the left atrial pressure cycle and the electrocardiogram may be seen in Fig. 3. Records were made daily after conversion until an " $a$ " wave appeared in the ADC, and in one case at weekly intervals up to three weeks.

In two patients the pressure pulses from the left and right atria were recorded through cardiac catheters introduced before the conversion.

The conversion was carried out during brief general anaesthesia using the Lown "Cardioverter" (American Optical Company).

\section{RESULTS}

One of the patients studied by cardiac catheterization had idiopathic atrial fibrillation, and an " $a$ " wave appeared in the left atrial pressure pulse as soon as sinus rhythm was restored. The other had chronic rheumatic mitral disease, and an "a" wave failed to appear on the left atrial pulse, even though it returned to the right atrial pressure pulse.

Evidence of mechanical left atrial activity, in the form of an " $a$ " wave on the left ventricular apex displacement curve (ADC), appeared immediately after conversion in five of the patients studied by this method.

In six of the remaining patients the immediate appearance of a jugular " $a$ " wave signalled the return of right atrial mechanical activity, but three to six days passed before the left atrial " $a$ " wave was detected on the ADC.

In one case the " $a$ " wave failed to return to the ADC during the observation period of three weeks, though an "a" wave reappeared immediately on the jugular venous pulse.

Of the seven patients with delayed return of the " a" wave, six had chronic rheumatic heart disease and one had ischaemic heart disease.

\section{Discussion}

Before this study was made we had encountered one patient with cerebral embolism occurring 36 


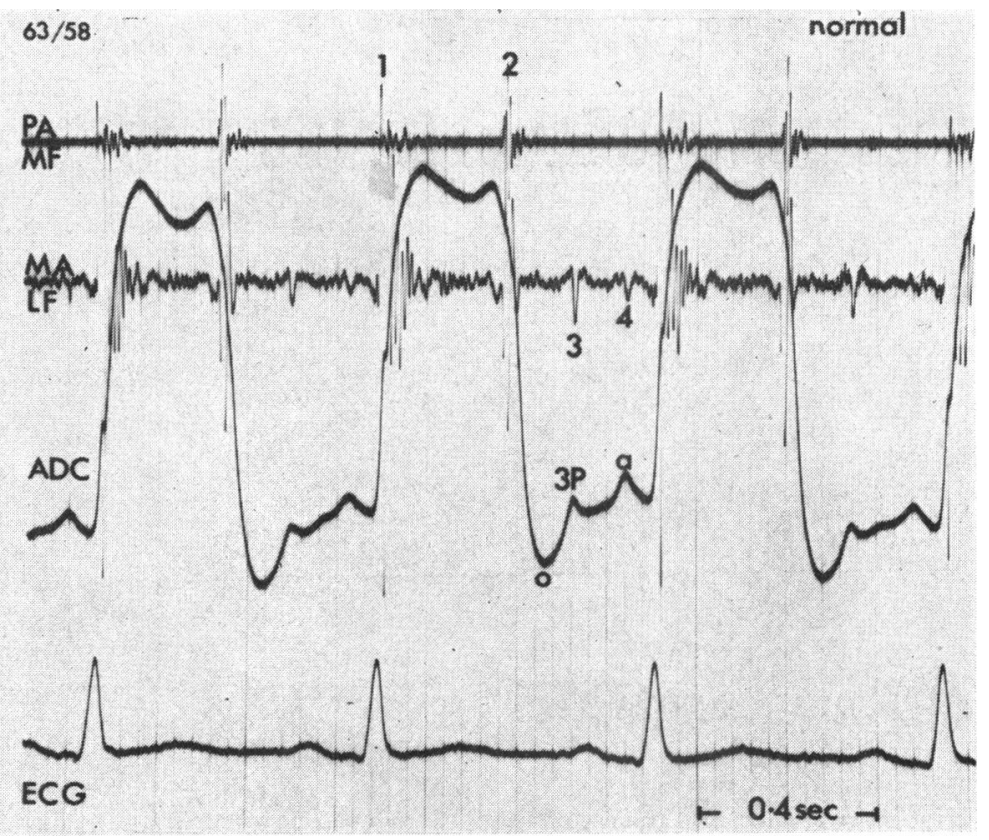

Fig. 1.-Apex displacement curve (ADC) of the left ventricle in a normal person showing the normal displacement wave due to left atrial contraction (" $a$ " wave). a, atrial contraction wave; $3 P$, third sound peak: the portion of the apex displacement curve written at the time of rapid ventricular filling and the third heart sound; $O$, point at which the mitral valve opens; PA, pulmonary area; MA, mitral area; MF, medium frequency phonocardiogram; LF, low frequency phonocardiogram; 1 , first heart sound; 2 , second heart sound; 3, third heart sound; and 4, fourth heart sound.

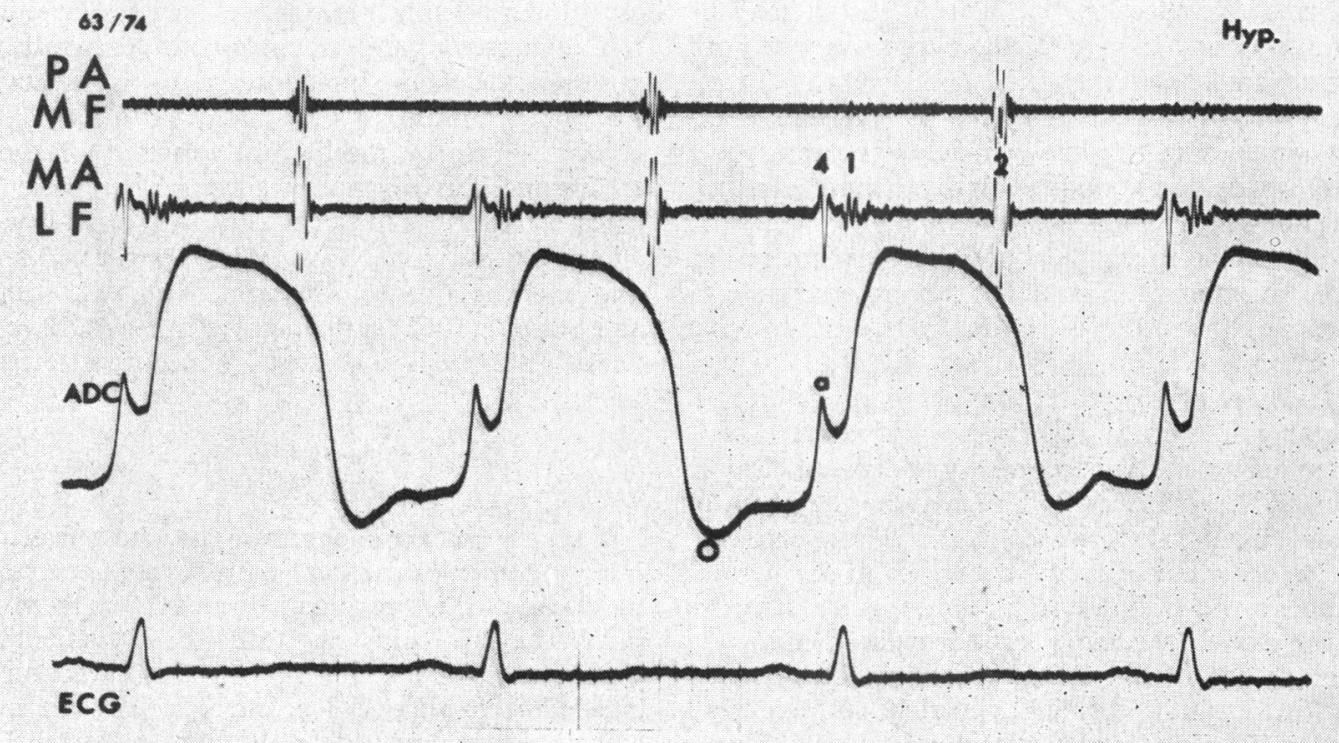

FIG. 2.-Apex displacement curve of the left ventricle in a patient with systemic hypertension showing the pathologically large "a" wave due to powerful atrial systole. Abbreviations as in Fig 1. 


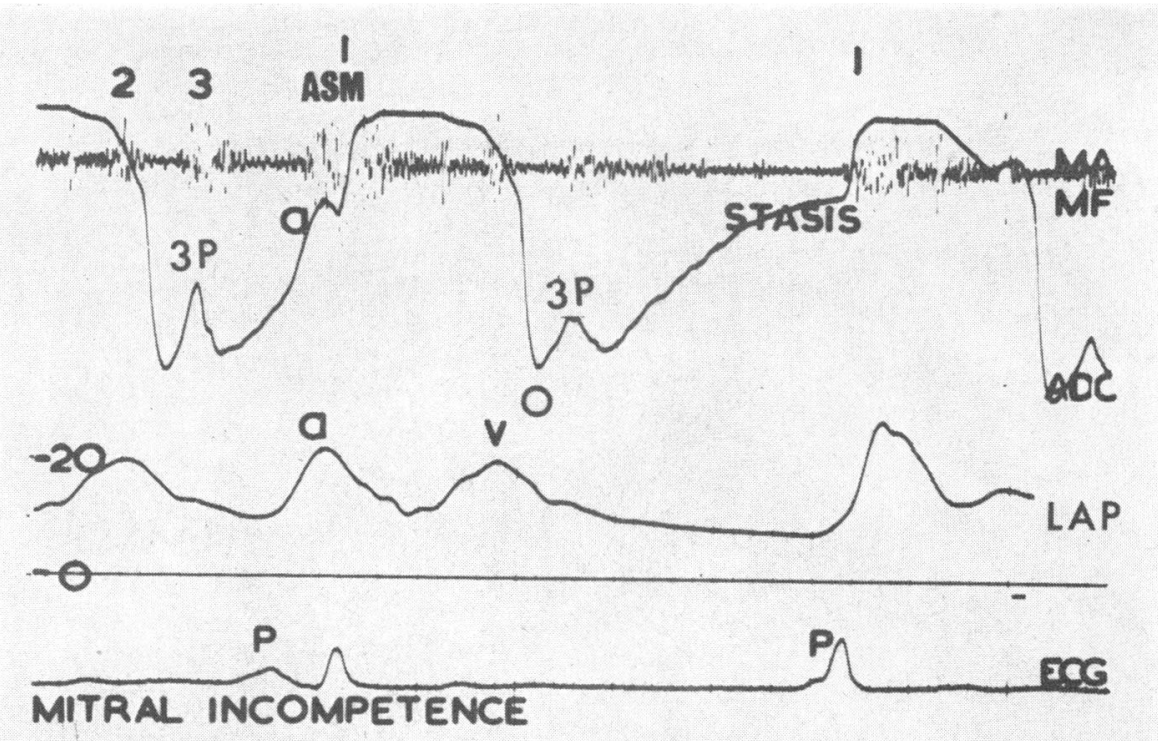

FIG. 3.-Left ventricular apex displacement curve (ADC) in a patient with mitral valve disease showing the absence of atrial contraction on the ADC and left atrial pressure curve (LAP) when a nodal beat occurs. The atrial systolic murmur (ASM) is also absent. Abbreviations as in Fig. 1.

hours after conversion from atrial fibrillation to sinus rhythm, and two patients in whom this occurred after four days. It is probable that the embolism occurred when the left atrium began to contract effectively. In order to minimize the risk from this complication, we believe that it may be desirable to continue anticoagulant treatment until the mechanical activity of the left atrium is detectable. The displacement curve of the skin overlying the apex of the left ventricle appears to be a suitable bedside technique for detecting the return of the effective left atrial contraction.

If detectable mechanical activity does not return after conversion it is probably not worth while to repeat the shock when the patient relapses into atrial fibrillation.

It is noteworthy that the delay or failure of detectable left atrial activity to return after conversion occurred most frequently in cases of chronic rheumatic heart disease. This may be the result of chronic rheumatic disease of the left atrial wall. The immediate return of " $a$ " waves to the jugular tracing or the right atrial pressure pulse suggests that the disease tends to spare the right atrium.

The return of function to the right atrium may boost the output of the right ventricle. If left atrial function does not return simultaneously a disproportion between the enhanced right ventricular output and the unchanged left ventricular output may occur. If other circumstances such as the left atrial pressure and the permeability of the pulmonary capillaries are of a certain level, this disproportion between the output of the right and left hearts may cause pulmonary oedema. We suggest that this mechanism may have been responsible for the unexpected and unexplained post-conversion pulmonary oedema encountered by Resnekov and McDonald (1965).

The failure of mechanical function of the left atrium in sinus rhythm in chronic rheumatic heart disease may be a distinct cause of disability, and Bramwell and Jones (1944) have suggested that it may even be a cause of death. Mitchell, Gilmore, and Sarnoff (1962) and Braunwald (1964), have supported Bramwell and Jones' concept of left atrial failure.

\section{SUMMARY}

The displacement curve of the chest wall overlying the apex of the left ventricle has been studied in 12 patients before and after conversion from atrial fibrillation to sinus rhythm. In 6 patients the return of a detectable left atrial contraction was delayed from three to six days, and in one mechanical left atrial activity could not be detected even after three weeks.

It is suggested that a clot may be discharged from 
the left atrium when it first resumes contraction, and that anticoagulant treatment should perhaps be continued until an " $a$ " wave appears on the apex displacement curve. Reference is made to the concept of left atrial failure with sinus rhythm in chronic rheumatic heart disease.

The authors wish to express their gratitude to the Clinical Research Sub-Committee and the Board of Governors of Charing Cross Hospital for making this work possible. Dr. T. Arcan was the holder of a British Council Fellowship.

They wish to thank Miss M. Alexander, Mr. H. Ketteringham, and Mr. Ariffin bin Musa for technical assistance.

\section{REFERENCES}

Bramwell, C., and Jones, A. M. (1944). Acute left auricular failure. Brit. Heart f., 6, 129.

Braunwald, E. (1964). Hemodynamic significance of atrial systole. Amer. F. Med., 37, 665.
Goldman, M. J. (1960). The management of chronic atrial fibrillation: indications for and method of conversion to sinus rhythm. Progr. cardiovasc. Dis., 2, 465.

Kory, R. C., and Meneely, G. R. (1951). Cardiac output in auricular fibrillation with observations on the effects of conversion to normal sinus rhythm. $\mathcal{F}$. clin. Invest., 30, 653.

Logan, W. F. W. E., Rowlands, D. J., Howitt, G., and Holmes, A. M. (1965). Left atrial activity following cardioversion. Lancet, $2,471$.

Lown, B., Amarasingham, R., and Neuman, J. (1962). New method for terminating cardiac arrhythmias. Use of synchronized capacitor discharge. $\mathcal{F}$. Amer. med. Ass., $182,548$.

Mitchell, J. H., Gilmore, J. P., and Sarnoff, S. J. (1962). The transport function of the atrium. Factors influencing the relation between mean left atrial pressure and left ventricular end diastolic pressure. Amer. F. Cardiol., 9, 237.

Nixon, P. G. F., Hepburn, F., and Ikram, H. (1964). Simultaneous recording of heart pulses and sounds. Brit. med. F., 1, 1169.

Resnekov, L., and McDonald, L. (1965). Pulmonary oedema following treatment of arrhythmias by direct-current shock. Lancet, 1, 506. 